\title{
Avaliação da completude da variável raça/cor nos sistemas nacionais de informação em saúde para aferição da equidade étnico-racial em indicadores usados pelo Índice de Desempenho do Sistema Único de Saúde
}

Evaluation of the race/color variable completeness in the national health information systems for the measuring of ethnic-racial inequality in indicators used by the Performance Index of the Brazilian Unified Health System

Rui Moreira Braz', Paulo de Tarso Ribeiro de Oliveira², Afonso Texeira dos Reis ${ }^{3}$, Nadia Maria da Silva Machado ${ }^{4}$

${ }^{1}$ Doutor em Medicina Tropical pela Universidade de Brasília (UnB) - Brasília (DF), Brasil. Administrador-Sanitarista no Departamento de Monitoramento e Avaliação do Sistema Único de Saúde, Secretaria Executiva, Ministério da Saúde - Brasília (DF), Brasil.

rui.braz@saude.gov.br

2 Doutor em Saúde Pública pela Escola Nacional de Saúde Pública (ENSP/ FIOCRUZ) - Rio de Janeiro (RJ), Brasil. Professor da Universidade Federal do Pará (UFPA) - Belém (PA), Brasil. Diretor do Departamento de Monitoramento e Avaliação do Sistema Único de Saúde, Secretaria Executiva, Ministério da Saúde - Brasília (DF), Brasil.

paulo.oliveira@saude.gov.br

${ }^{3}$ Mestre em Saúde Pública pela Universidade Federal de Minas Gerais (UFMG) - Belo Horizonte (MG), Brasil. Coordenador Geral de Monitoramento e Avaliação, Departamento de Monitoramento e Avaliação do Sistema Único de Saúde, Secretaria Executiva, Ministério da Saúde - Brasília (DF), Brasi afonso.reis@saude.gov.br

${ }^{4}$ Especialista em Gestão de Políticas Sociais pela Universidade Paulista (UNIP) -Brasília (DF), Brasil. Consultora da OPAS/OMS no Departamento de Monitoramento e Avaliação do Sistema Único de Saúde, Secretaria Executiva, Ministério da Saúde - Brasília (DF), Brasil. nadia.machado@saude.gov.br
RESUMO: Avaliou-se o preenchimento da variável raça/cor em oito sistemas/módulos nacionais de informação em saúde, bem como a viabilidade de cálculo e uso de indicadores segundo esse quesito na avalição do Sistema Único de Saúde. Somente três sistemas/ módulos estavam adequados para validar três dos 24 indicadores usados pelo Índice de Desempenho do SUS. Apesar dos avanços nas estratégias para saúde de grupos étnico-raciais, o aprimoramento do preenchimento da variável permitirá melhor avaliação da situação de saúde da população negra. Recomenda-se monitoramento dessa variável por meio da qualificação da coleta e alimentação dos dados. É importante socializar o debate sobre equidade étnico-racial em saúde, utilizando-se o referido índice de desempenho para acompanhamento da atenção à saúde da população negra pelos governos e movimentos sociais.

PALAVRAS-CHAVE: Completude; Avaliação de desempenho; População negra

ABSTRACT: The race/color variable was assessed in eight national systems/modules of health information. The calculation feasibility and use of indicators were also evaluated as for this same issue under the Unified Health System. Only three modules/systems could validate three out of the 24 indicators used by the SUS Performance Index. Despite advances towards health of ethnic and racial groups, the completeness of the variable is mandatory to allow for a better evaluation of the black population heath conditions. The monitoring of this variable is recommended by improving data collection and feed. It is important to broaden the debate on ethnic and racial equity in health by applying the mentioned performance index for monitoring the black population health care by Governments and social movements.

KEYWORDS: Completeness; Performance assessment; Black population. 


\section{Introdução}

A busca de conteúdo informacional aprimorado e capaz de incorporar as múltiplas dimensôes das iniquidades em saúde em diferentes contextos sociais representa um desafio (MAGALHÁES, 2007). No Brasil, o Ministério da Saúde vem fortalecendo a agenda de promoção da equidade racial em saúde com a implementação de diversas estratégias para identificar, prevenir e enfrentar as desigualdades e discriminaçóes de segmentos excluídos historicamente. Em 2009, foi instituída a Política Nacional de Saúde Integral da População Negra (PNSIPN), cujo objetivo geral é promover a saúde integral dessa população, priorizando a redução das desigualdades étnico-raciais, o combate ao racismo e à discriminação nas instituiçôes e serviços do Sistema Único de Saúde (SUS) para redução das iniquidades (BRASIL, 2009). As diretrizes e metas para alcance desse objetivo encontram-se ratificadas no Plano Nacional de Saúde 2012-2015 (BRASIL, 2011a, p. 70). No processo de implantação dessa Política, o MS tem garantido a gestão transparente e participativa com os diversos atores sociais, possibilitando o acompanhamento dos resultados com recorte étnico-racial por meio dos grandes sistemas de informação em saúde, a exemplo do que ocorre atualmente com a AIDS, Tuberculose e Hanseníase, na Sala de Apoio à Gestão Estratégica (SAGE), disponível em: <http://189.28.128.178/sage/>.

A qualidade dos sistemas de vigilância em saúde é definida por "atributos como a completude, oportunidade, utilidade, sensibilidade, especificidade, valor preditivo positivo, simplicidade, flexibilidade, aceitabilidade e representatividade" (WORLD HEALTH ORGANIZATION, 2006, p.15). A completude dos dados influencia os demais atributos, pois a qualidade da informação é fundamental para avaliar o desempenho dos sistemas de saúde. A completude se refere à proporção de casos notificados, apropriadamente, para as autoridades de saúde, sendo importante para doenças de ocorrência frequente e necessária para comparaçôes nos âmbitos nacional e internacional (DOYLE et al, 2002). É, portanto, a relação entre o número de casos registrados e ocorridos, mantendo conexão com a sensibilidade do sistema e assegurando se os dados foram coletados e registrados adequadamente, inclusive para os eventos vitais como nascimentos e óbitos.

Alguns estudos analisaram a completude dos sistemas de informação, seja para eventos específicos, como tuberculose (MOREIRA, MACIEL, 2008), mortalidade por suicídio (MACENTE; ZONDANADE, 2010), declaração de nascidos vivos e óbito neonatal (BARBUSCIA; RODRIGUES-JÚNIOR, 2011) ou para vigilância de grupos de doenças nos Estados Unidos da América (JAJOSKY; GROSECLOSE, 2004), no Colorado-EUA (VOGT, et al., 2006) ou na Coréia (YOO, et al., 2009). Avaliar os sistemas de informação é imprescindível, pois são instrumentos para o diagnóstico situacional, podendo caracterizar populaçóes sob risco e planejar estratégias conforme as especificidades de cada grupo populacional (ZILLMER, et al., 2010). Os sistemas de informação podem contribuir, ainda, para práticas de saúde baseadas em evidências (RODRIGUES, 2000). A apropriação das informaçóes em saúde pelos gestores e pela sociedade deve considerar os pontos fortes e suas limitaçóes, resultado de avaliaçôes regulares e sistemáticas dos dados disponibilizados. Nesse sentido, a qualidade da informação é essencial para a análise da situação sanitária e para a programação de açóes de saúde (LIMA, et al., 2009), tornando os sistemas de informação primordiais nos processos de planejamento, monitoramento, avaliação e tomada de decisão nos distintos níveis de competência das políticas públicas de saúde (LAGUARDIA et al., 2004).

O Índice de Desempenho do SUS (IDSUS) é um programa instituído pelo MS com o objetivo principal de avaliar o desempenho do SUS quanto ao cumprimento de seus princípios da universalidade do acesso, integralidade da atenção, regionalização e hierarquização; nos municípios, regióes, estados e no Brasil, tem como objeto o 'SUS que atende o residente em cada município brasileiro' e, como linhas avaliativas, as dificuldades do acesso e os resultados segundo as melhores respostas esperadas. Objetiva, também, detectar deficiências, visando à implementação de melhorias e não apenas à classificação dos níveis de desempenho (BRASIL, 2011b). No cálculo do IDSUS, são utilizadas as notificaçôes de casos de doenças, internações, atendimentos ambulatoriais e eventos vitais constantes dos 
diversos sistemas de informação utilizados pelos três níveis de gestão (municipal, estadual e federal). Visando a melhorar a avaliação das equidades entre grupos populacionais, o MS propôs avaliar a inclusão de recorte étnico-racial nos indicadores do IDSUS com vistas à melhoria das informaçôes voltadas para a política nacional de saúde integral da população negra. Para que o IDSUS represente a realidade desse grupo populacional, é necessário que a coleta de dados relativos à raça/cor seja qualificada para não fragilizar a construção de indicadores que melhor avaliem a atenção à saúde quando se trata de um segmento específico. Nessa perspectiva, este estudo objetiva analisar a completude do campo raça/ cor em oito sistemas/módulos de informação em saúde para verificar a possibilidade de aferição da equidade étnico-racial nos indicadores usados pelo IDSUS.

\section{Método}

Trata-se de estudo de abrangência nacional, compreendendo todos os municípios e estados que alimentam os sistemas de informação objeto desta avaliação. Foram analisados os seguintes sistemas de informação: 1) Sistema de Informações sobre Nascidos Vivos (SINASC); 2) Sistema de Informações de Agravos de Notificação (SINAN) - módulos: a) Sífilis congênita; b) Hanseníase; c) Tuberculose; 3) Sistema de Informaçóes Ambulatoriais do Sistema Único de Saúde (SIA/SUS) - módulos: a) Autorização de Procedimentos Ambulatoriais de Alta Complexidade (APAC); b) Boletim de Produção Ambulatorial Individualizado (SIA-BPAI); 4) Sistema de Informaçôes Hospitalares (SIH/SUS); 5) Sistema de Informaçôes sobre Mortalidade (SIM). De acordo com o percentual de preenchimento do campo raça/ cor, os sistemas de informação foram classificados em: adequado ( $\geq 90 \%$ de preenchimento) ou inadequado (<90\% de preenchimento), em relação à possibilidade de cálculo dos indicadores do IDUS, com recorte étnico-racial. Para que o sistema fosse considerado adequado, cada um dos três anos mais recentes deveria apresentar pelo menos $90 \%$ de preenchimento do campo raça/cor. Foram considerados somente os três anos mais recentes disponíveis em cada série histórica por ser este o período definido para construção dos indicadores do IDSUS. Os demais anos anteriores foram utilizados para análise da evolução temporal do preenchimento do campo avaliado.

A possibilidade de validação com recorte étnico-racial dos 24 indicadores do IDSUS foi verificada adotando-se dois critérios: 1) com possibilidade de validação - quando o indicador tinha como fonte de dados um dos sistemas de informaçáo avaliado como adequado, segundo a classificação acima; 2) sem possibilidade de validação - quando o indicador teve como fonte de dados um dos sistemas de informação avaliado como inadequado, ou, ainda, quando o indicador não utilizava, como fonte de dados, os sistemas de informação avaliados.

A completude da variável foi avaliada utilizando-se os programas de tabulação de dados de saúde (TABWIN e TABNET) do Departamento de Informática do SUS (Datasus), Secretaria de Gestão Estratégica e Participativa, Ministério da Saúde. Todos os dados utilizados são públicos e disponibilizados na internet pelo Datasus em <http://www2.datasus.gov.br/ DATASUS/index.php?area=02> (dados do SINAN) e <http://msbbs.datasus.gov.br/default.htm> (demais dados). Por envolver apenas o uso de dados secundários agregados, sem identificação de indivíduos, este estudo não acarretou risco às pessoas ou aos animais, dispensando os requisitos da Resolução 196/1996, do Conselho Nacional de Saúde.

\section{Resultados}

Os resultados apurados para o SINASC demonstraram a disponibilidade de uma série histórica de nascidos vivos de 1994 até 2012. O preenchimento do campo raça/cor teve início em 1996 com um percentual de 1,3\%, alcançando 95,6\% em 2010. Quando analisados somente os três anos mais recentes, de 2010 a 2012, verificou-se que em 2011 o percentual de preenchimento desse campo teve queda vertiginosa para $41,1 \%$, sendo maior em 2012, quando decaiu para 3,5\%. (Figura 1A). A série histórica de Sífilis congênita estava disponível no SINAN no período de 2001 a 2010. 
Figura 1. Percentual de preenchimento do campo raça/cor em oito sistemas/módulos de informação do SUS, em diferentes períodos. Brasil, 1994 a 2012
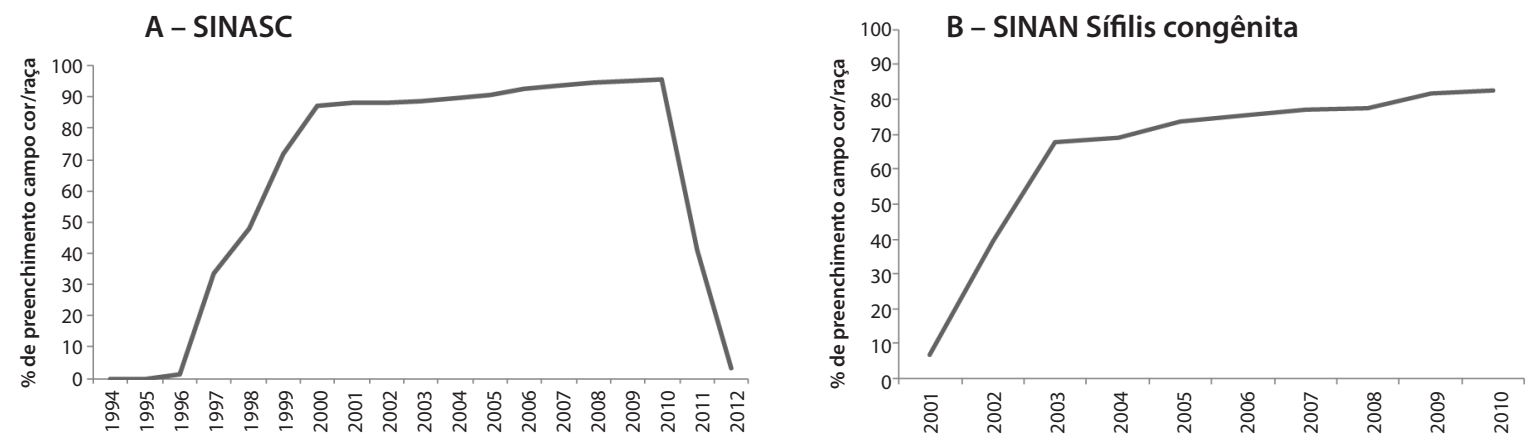

\section{C - SINAN Hanseníase}
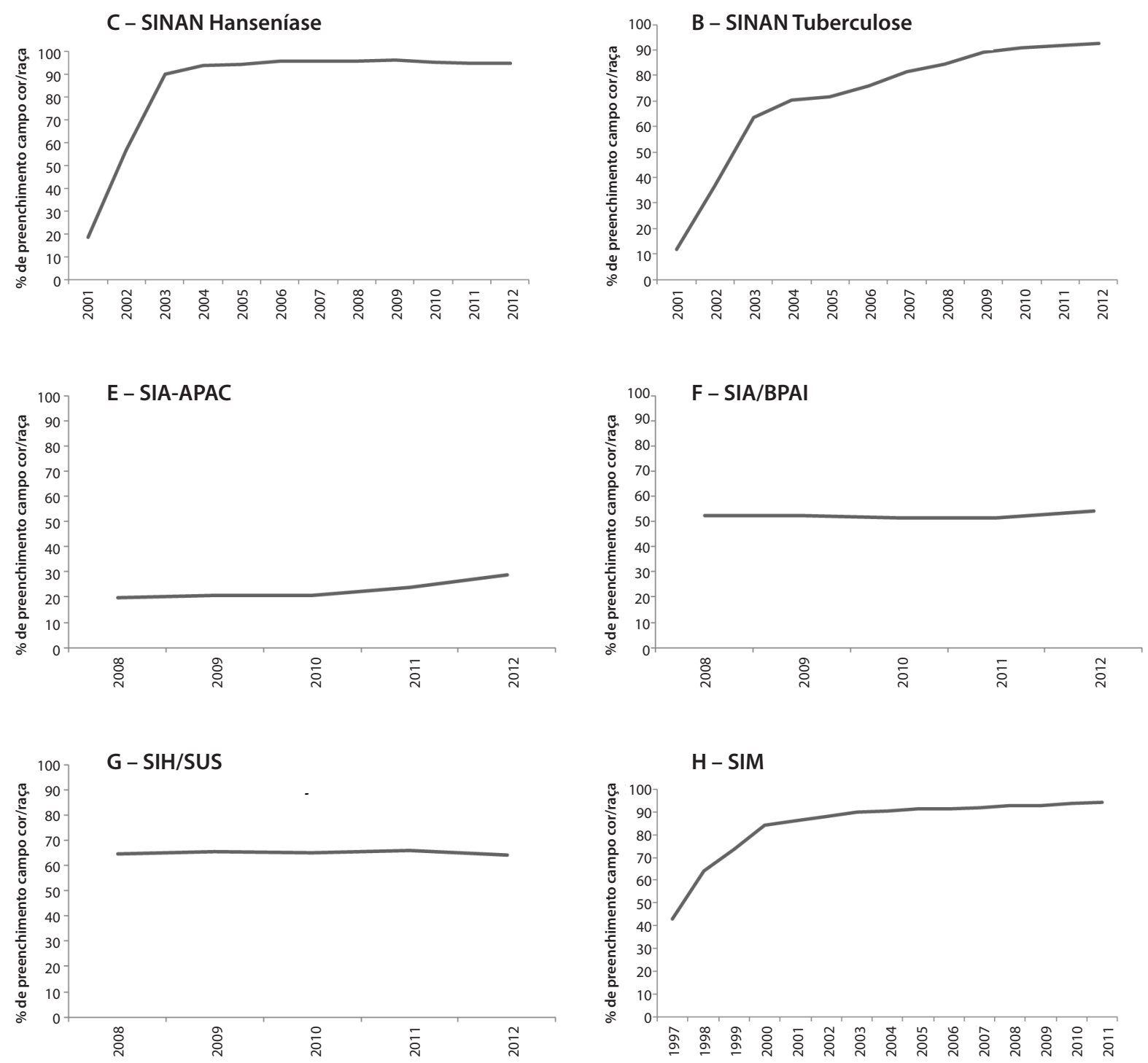
Tabela 1. Classificação de oito sistemas/módulos de informação do SUS em relação à completude do campo raça/cor. Brasil, 2008 a 2012

\begin{tabular}{|l|c|c|c|}
\hline $\begin{array}{l}\text { SISTEMA/MÓDULO DE } \\
\text { INFORMAÇÃO }\end{array}$ & $\begin{array}{c}\text { PERÍODO TRÊS ANOS } \\
\text { MAIS RECENTES }\end{array}$ & $\begin{array}{c}\text { \% PREENCHIMENTO } \\
\text { CAMPO RAÇA/COR } \\
\text { (MÉDIA TRÊS ANOS) }\end{array}$ & CLASSIFICAÇÃO \\
\hline SINASC & $2010-2012$ & 46,7 & Inadequado \\
\hline SINAN-Síflis congênita & $2008-2010$ & 80,6 & Inadequado \\
\hline SINAN-Hanseníase & $2010-2012$ & 94,8 & Adequado \\
\hline SINAN-Tuberculose & $2010-2012$ & 91,7 & Adequado \\
\hline SIA/SUS-APAC & $2010-2012$ & 24,4 & Inadequado \\
\hline SIA/SUS-BPAI & $2010-2013$ & 52,5 & Inadequado \\
\hline SIH-SUS & $2010-2012$ & 65,1 & Inadequado \\
\hline SIM & $2009-2011$ & 93,5 & Adequado \\
\hline
\end{tabular}

Fonte: MS/SVS/SAS - SINASC, SINAN, SIA/SUS, SIH/SUS e SIM.

Nesse período, observou-se incremento contínuo do percentual de preenchimento do campo raça/cor para essa doença, iniciando em 2001 com 6,8\% de preenchimento, passando para $82,6 \%$ em 2010. Em todos os anos, o percentual de preenchimento do campo raça/ cor manteve-se abaixo de 90\% (Figura 1B).

Os casos de Hanseníase estão disponíveis para análise no período de 2001 a 2012. O percentual de preenchimento do campo raça/cor iniciou com $18,6 \%$, em 2001, e ultrapassou 90\% em 2004, mantendo-se acima desse valor nos anos seguintes. Nos três anos mais recentes da série, 2010 a 2012, o percentual de preenchimento desse campo variou entre 94,6\% e 95,1\% (Figura 1C). A série histórica dos casos de Tuberculose abrange o período de 2001 a 2012. O preenchimento do campo raça/ cor iniciou-se em 2001, com percentual de 11,8\%. Apesar de o preenchimento desse campo ter aumentado gradualmente durante todo o período, somente ultrapassou os $90 \%$ em 2010, mantendo-se acima desse referencial no período de 2010 a 2012 (Figura 1D).

Em relação ao módulo de autorização de procedimentos ambulatoriais de alta complexidade - SIA/ APAC, não houve muita variação no percentual de preenchimento do campo raça/cor, analisado no período de 2008 a 2012. O preenchimento desse campo manteve-se abaixo dos 30\% em todos os anos, variando de 19,6\% em 2008 a 28,8\% em 2012 (Figura 1E). O percentual de preenchimento do campo raça/cor no Boletim de Produção Ambulatorial Individualizada - SIA/SUS-BPAI também apresentou pouca variação no período de 2008 a 2011. O preenchimento do campo manteve-se um pouco acima de $50 \%$ em todos os anos, variando de 52,4\%, em 2008, a 54,2\%, em 2012 (Figura 1F).

No sistema de internaçóes hospitalares - SIH/ SUS, o percentual de preenchimento do campo raça/ cor apresentou-se estável no período de 2008 a 2012, com valores variando entre $64,3 \%$, em 2012 , e $65,9 \%$, em 2011 (Figura 1G). Os dados temporais sobre mortalidade - SIM - foram analisados no período de 1997 a 2010. Nesse período, o percentual de preenchimento do campo raça/cor iniciou-se com 43,2\%, em 1997, e apresentou incremento progressivo até alcançar 90,6\% em 2004, mantendo-se acima dos $90 \%$ no restante do período. Nos três anos mais recentes, 2009 a 2011, o percentual de preenchimento desse campo variou de $92,8 \%$ a $94,0 \%$ (Figura $1 \mathrm{H}$ ). 
A avaliação da completude do campo raça/cor dos oito sistemas/módulos de informação em saúde, voltada para a viabilidade de recorte étnico-racial dos indicadores do IDSUS, permitiu classificar três $(37,5 \%)$ sistemas/módulos como adequados e cinco $(62,5 \%)$ como inadequados (Tabela 1). Foi possível verificar, também, que, dos 24 indicadores utilizados para construção do IDSUS, apenas três $(12,5 \%)$ foram considerados como tendo possibilidade de validação em relação ao campo raça/cor: 1) Proporção de acesso hospitalar dos óbitos por acidente; 2) Proporção de cura de casos novos de tuberculose pulmonar bacilífera; e 3) Proporção de cura dos casos novos de hanseníase. Os demais indicadores (87.5\%) foram considerados como sem possibilidade de validação por apresentarem menos de $90 \%$ de preenchimento do campo raça/cor (Tabela 2).

\section{Discussão}

Enfatiza-se, inicialmente, que o propósito aqui não foi avaliar a qualidade dos sistemas de informação em saúde contidos neste estudo, porquanto é sabido da importância desses sistemas no direcionamento das açôes de atenção à saúde no SUS. Pretendeu-se verificar, concretamente, a possibilidade de aferição da equidade étnico-racial no SUS - conceito que considera as desigualdades sociais como injustas e evitáveis (BRASIL, 2013). Nesse sentido, a presente avaliação de completude demonstrou que a maioria dos sistemas de informação estudados não possibilitou a validação dos indicadores do IDSUS em relação ao atributo raça/cor. Isso implica a dificuldade atual de se dimensionar o desempenho do SUS em relação ao princípio da equidade da atenção à saúde com recorte étnico-racial. Especificamente, daquele grupo alcançado pela Política Nacional de Saúde Integral da População Negra.

Alguns sistemas de informação - como SIM, SINAN-Hanseníase e SINAN-Tuberculose - mostraram-se adequados, enquanto os demais sistemas apresentaram limitaçóes evidentes quanto ao preenchimento do campo raça/cor. Os achados do presente estudo são corroborados por Soares-Filho (2012), que considerou satisfatória a proporção de, no mínimo, 90\% de preenchimento do campo raça/cor ao analisar a completude dessa variável nos sistema de informação SIM, SINASC e SINAN (módulos AIDS, hanseníase, tuberculose, doença de chagas e sífilis em gestantes). Por outro lado, nos demais módulos do SINAN e no SIH/SUS, o preenchimento do atributo raça/cor não foi satisfatório. Felix et al. (2012), em um estudo sobre mortalidade por câncer de mama, categorizaram como excelente e bom o fato de a variável raça/cor, na declaração de óbito, ter apresentado até $10 \%$ de preenchimento incompleto, ou seja, um mínimo de $90 \%$ de preenchimento. O mesmo critério foi utilizado por Macente e Zandonade (2010) ao avaliarem a mortalidade por suicídio no SIM.

Um aspecto a ser observado em relação ao SINASC é que houve um declínio abrupto no preenchimento do campo raça/cor nos anos de 2011 e 2012, diferentemente dos anos anteriores, quando esse preenchimento foi considerado satisfatório, tanto nas análises de Soares-Filho (2012) quanto neste estudo (Figura 1A). Esse fato poderá inviabilizar o cálculo de um dos indicadores do IDSUS - Proporção de nascidos vivos de mães com sete ou mais consultas de pré-natal - em relação ao enfoque étnico-racial (Tabela 2). A introdução do recorte étnico-racial no cálculo do IDSUS é importante, porque esse índice pode melhorar a pontuação de desempenho do SUS naqueles entes federados que apresentarem melhor execução da Política Nacional de Saúde Integral da População Negra. Contrariamente, aqueles com baixo desempenho alcançarão menores notas. Este aspecto é primordial no direcionamento das políticas públicas de saúde. Por isso, é relevante a qualificação do campo raça/ cor nos grandes sistemas nacionais de informação em saúde para identificar as diferenças e as necessidades reais, possibilitando melhor acerto nas políticas de promoção da equidade. Ressalta-se que, apesar do entendimento de que não se estabelece uma relação de causalidade entre o quesito raça/cor e o surgimento de doenças, essa informação pode dar significativas indicações sobre as condiçôes de vida e saúde da população negra (APARECIDA-PINTO; SOUZAS, 2002).

Deve-se salientar a importância do IDSUS não só para mensurar as desigualdades e classificar as instâncias de gestão no SUS, mas, principalmente, para detectar as deficiências e subsidiar o planejamento das 
Tabela 2. Indicadores do Índice de Desempenho do Sistema Único de Saúde segundo fonte de dados e possibilidade de validação do indicador a partir do campo raça/cor. Brasil, 2013

\begin{tabular}{|c|c|c|c|}
\hline No & INDICADOR & $\begin{array}{l}\text { FONTE DE } \\
\text { DADOS }\end{array}$ & $\begin{array}{l}\text { POSSIBILIDADE } \\
\text { DE VALIDAÇÃO } \\
\text { DO INDICADOR }\end{array}$ \\
\hline 1 & Cobertura populacional estimada pelas equipes básicas de saúde & CNES e IBGE & Sem possibilidade \\
\hline 2 & Cobertura populacional estimada pelas equipes básicas de saúde bucal & CNES e IBGE & Sem possibilidade \\
\hline 3 & $\begin{array}{l}\text { Proporção nascidos vivos de mães com no mínimo sete consultas de pré- } \\
\text { natal }\end{array}$ & SINASC & Sem possibilidade \\
\hline 4 & $\begin{array}{l}\text { Razão de exames de mamografia de rastreamento realizados em mulheres } \\
\text { de } 50 \text { a } 69 \text { e população da mesma faixa etária. }\end{array}$ & SIA-BPAI & Sem possibilidade \\
\hline 5 & $\begin{array}{l}\text { Razão exames citopatológicos do colo do útero em mulheres de } 25 \text { a } 59 \\
\text { anos e população da mesma faixa etária. }\end{array}$ & SIA-BPAI & Sem possibilidade \\
\hline 6 & $\begin{array}{l}\text { Razão de procedimentos ambulatoriais selecionados de média } \\
\text { complexidade e população residente. }\end{array}$ & SIA/SUS e IBGE & Sem possibilidade \\
\hline 7 & $\begin{array}{l}\text { Razão de internações clínico-cirúrgicas de média complexidade e população } \\
\text { residente. }\end{array}$ & $\mathrm{SIH} / \mathrm{SUS}$ e IBGE & Sem possibilidade \\
\hline 8 & $\begin{array}{l}\text { Razão de procedimentos ambulatoriais de alta complexidade sele-cionados } \\
\text { e população residente. }\end{array}$ & SIA/SUS e IBGE & Sem possibilidade \\
\hline 9 & $\begin{array}{l}\text { Razão de internações clínico-cirúrgicas de alta complexidade e população } \\
\text { residente. }\end{array}$ & $\mathrm{SIH} / \mathrm{SUS}$ e IBGE & Sem possibilidade \\
\hline 10 & $\begin{array}{l}\text { Proporção de procedimentos ambulatoriais de média complexidade para } \\
\text { não residentes. }\end{array}$ & SIA/SUS & Sem possibilidade \\
\hline 11 & $\begin{array}{l}\text { Proporção de internações de média complexidade realizadas para não } \\
\text { residentes. }\end{array}$ & $\mathrm{SIH} / \mathrm{SUS}$ & Sem possibilidade \\
\hline 12 & $\begin{array}{l}\text { Proporção de procedimentos ambulatoriais de alta complexidade realizados } \\
\text { para não residentes. }\end{array}$ & SIA/SUS & Sem possibilidade \\
\hline 13 & Proporção de internações de alta complexidade para não residentes. & $\mathrm{SIH} / \mathrm{SUS}$ & Sem possibilidade \\
\hline 14 & Proporção de acesso hospitalar dos óbitos por acidente. & SIM & $\begin{array}{l}\text { Com } \\
\text { possibilidade }\end{array}$ \\
\hline 15 & Cobertura com a vacina tetravalente. & SI-PNI e SINASC & Sem possibilidade \\
\hline 16 & Proporção de cura de casos novos de tuberculose pulmonar bacilífera. & $\begin{array}{c}\text { SINAN- } \\
\text { TUBERCULOSE }\end{array}$ & $\begin{array}{c}\text { Com } \\
\text { possibilidade }\end{array}$ \\
\hline 17 & Proporção de cura dos casos novos de hanseníase. & $\begin{array}{c}\text { SINAN- } \\
\text { HANSENÍASE }\end{array}$ & $\begin{array}{c}\text { Com } \\
\text { possibilidade }\end{array}$ \\
\hline 18 & Taxa de Incidência de Sífilis Congênita. & $\begin{array}{l}\text { SINAN-SÍFILIS } \\
\text { CONG e SINASC }\end{array}$ & Sem possibilidade \\
\hline 19 & Proporção de internações sensíveis à atenção básica (ISAB). & $\mathrm{SIH} / \mathrm{SUS}$ & Sem possibilidade \\
\hline 20 & Média da ação coletiva de escovação dental supervisionada. & SIA/SUS e IBGE & Sem possibilidade \\
\hline
\end{tabular}




\begin{tabular}{|c|l|c|c|}
\hline No & \multicolumn{1}{|c|}{ INDICADOR } & $\begin{array}{c}\text { FONTE DE } \\
\text { DADOS }\end{array}$ & $\begin{array}{c}\text { POSSIBILIDADE } \\
\text { DE VALIDAÇÃO } \\
\text { DO INDICADOR }\end{array}$ \\
\hline $\mathbf{2 1}$ & Proporção de exodontia em relação aos procedimentos. & SIA/SUS e IBGE & Sem possibilidade \\
\hline $\mathbf{2 2}$ & Proporção de parto normal. & SINASC & Sem possibilidade \\
\hline $\mathbf{2 3}$ & $\begin{array}{l}\text { Proporção de óbitos, em menores de 15 anos, nas Unidades de Terapia } \\
\text { Intensiva UTI. }\end{array}$ & SIH/SUS & Sem possibilidade \\
\hline $\mathbf{2 4}$ & Proporção de óbitos nas internações por infarto agudo do miocárdio (IAM). & SIH/SUS & Sem possibilidade \\
\hline
\end{tabular}

Fonte: Elaboração própria.

Notas: CCSAP: Condições Cardiológicas Sensíveis à Atenção Primária; HAS: Hipertensão Arterial Sistêmica; IC: Insuficiência Cardíaca.

ações para enfrentamentos das situaçóes étnico-raciais adversas. É certo que existem vários documentos que abordam estratégias relacionados à melhoria da saúde de grupos étnico-raciais, a exemplo da PNSIPN, denotando grandes avanços a serem considerados, pois se trata da construção de uma política setorial, com recorte racial, geradora de processo de diferenciação e de busca de legitimação, no âmbito da saúde pública, dos agravos sofridos pela populaçáo negra (MAIO; MONTEIRO, 2005). A existência de políticas avançadas no papel é um grande passo, mas não é suficiente para garantir avanços sociais de fato, tanto em relação aos impactos da política quanto em relação ao direcionamento de recursos e esforços governamentais, pois a não adoção de medidas estatais de combate às desigualdades raciais contribui para sua manutenção (FAUSTINO, 2012), principalmente, em sociedades como a brasileira, na qual relaçóes de classe são racializadas e relaçóes raciais são dependentes da classe social (CHOR; ARAUJO-LIMA, 2005). Para que o IDSUS possa ser o indutor das políticas de saúde com recorte étnico-racial, torna-se fundamental o preenchimento adequado do campo raça/cor nos sistemas de informação que fornecem dados para a construção de seus indicadores.
Torna-se necessária, ainda, a discussão permanente sobre o alcance de indicadores selecionados a partir de sua repercussão na saúde e, também, de sua capacidade de dar visibilidade às dinâmicas de interação de grupos populacionais específicos (MAGALHÃES, 2007).

A realidade demonstrada neste estudo evidenciou que ainda existe um longo caminho a se percorrer em relação ao preenchimento do campo raça/cor nos grandes sistemas nacionais de informação em saúde, de forma a possibilitar a adequada avaliação do SUS nas três esferas de gestão, com recorte étnico-racial. Em virtude dessa situação, recomenda-se que os gestores desenvolvam açóes para superar essa limitação, possibilitando melhores opçóes de acompanhamento da PNSIPN. Um aspecto importante é o monitoramento permanente do preenchimento da variável raça/cor nos sistemas de informação para detectar as fragilidades e propor capacitação para os responsáveis pela coleta e alimentaçáo dos dados. É importante, também, socializar o debate sobre o IDSUS referente à equidade étnico-racial em saúde, possibilitando aos movimentos sociais melhor acompanhamentos das políticas governamentais específicas.

\section{Referências}

APARECIDA-PINTO, E.; SOUZAS, R. Etnicidade e saúde da população negra no Brasil. Cadernos de Saúde Pública, Rio de Janeiro, v. 18, n. 5, p.1144-1145, set-out, 2002.
BARBUSCIA, D.M.; RODRIGUES-JÚNIOR, A. L. Completude da informação nas declarações de nascido vivo e nas declarações de óbito neonatal precoce e fetal, da região de Ribeirão Preto, São 
Paulo, Brasil, 2000-2007. Cadernos de Saúde Pública, Rio de Janeiro, v. 27, n. 6, p. 1192-1200, jun., 2011.

BRASIL. Ministério da Saúde. Política nacional de saúde integral da população negra. Anexo da Portaria GM N. 992, de 13 de maio de 2009. Brasília: Ministério da Saúde, 2009.

Ministério da Saúde. Secretaria Executiva. Plano Nacional de Saúde - PNS 2012-2015. Brasília: Ministério da Saúde, 2011 a.

Ministério da Saúde. Secretaria Executiva. Índice de desempenho do Sistema Único de Saúde - IDSUS. Brasília: Ministério da Saúde, 2011 b.

Ministério da Saúde. Secretaria Executiva. Política nacional de informação e informática em saúde - PNIIS. Brasília: Ministério da Saúde, 2013.

CHOR, D.; ARAUJO-LIMA, C. R. Aspectos epidemiológicos das desigualdades raciais em saúde no Brasil. Cadernos de Saúde Pública, Rio de Janeiro, v. 21, n. 5, p. 1586-1594, set-out, 2005.

DOYLE, T.J.; GLYNN, M.K.; GROSECLOSE, S.L. Completeness of notifiable infectious disease reporting in the United States: an analytical literature review. American Journal of Epidemiology, Baltimore, v. 155, n. 9, p.866-874, 2002.

FAUSTINO, D.M.O. A equidade racial nas políticas de saúde. In: BATISTA, L.E.; WERNECK, J.; LOPES, F. (Org). Saúde da população negra. Brasília: ABNP, 2012.

FELIX, J.D. et al. Avaliação da completude das variáveis epidemiológicas do Sistema de Informação sobre Mortalidade em mulheres com óbitos por câncer de mama na Região Sudeste - Brasil (1998 a 2007). Ciência \& Saúde Coletiva, Rio de Janeiro, v.17, n. 4, p. $945-953,2012$.

JAJOSKY, R.A.; GROSECLOSE, S.L. Evaluation of reporting timeliness of public health surveillance systems for infectious diseases. BMC Public Health, London, v. 4, n. 29, p. 1-9, 2004.

LAGUARDIA, J. et al. Sistema de Informação de Agravos de Notificação (Sinan): desafios no desenvolvimento de um sistema de informação em saúde. Epidemiologia e Serviços de Saúde, Brasília, v. 13, n. 3, p. 135-146, 2004.

LIMA, C.R.A. et al. Revisão das dimensões de qualidade dos dados e métodos aplicados na avaliação dos sistemas de informação em saúde. Cadernos de Saúde Pública, Rio de Janeiro, v. 25, n. 10, p. 2095-2109, out., 2009.
MACENTE, L.B.; ZANDONADE, E. Avaliação da completude do Sistema de Informação sobre Mortalidade por suicídio na região Sudeste, Brasil, no período de 1996 a 2007. Jornal Brasileiro de Psiquiatria, Rio de Janeiro, v. 59, n. 3, p. 173-181, 2010.

MAIO, M.C.; MONTEIRO, S. Tempos de racialização: o caso da 'saúde da população negra' no Brasil. História, Ciências, Saúde - Manguinhos, Rio de Janeiro, v. 12, n. 2, p. 419-446, maio-ago. 2005.

MAGALHÃES, R. Monitoramento das desigualdades sociais em saúde: significados e potencialidades das fontes de informação. Ciência \& Saúde Coletiva, Rio de Janeiro, v. 12, n. 3, p. 667-673, 2007.

MOREIRA, C.M.M.; MACIEL, E.L.N. Completude dos dados do Programa de Controle da Tuberculose no Sistema de Informação de Agravos de Notificação no Estado do Espírito Santo, Brasil: uma análise do período de 2001 a 2005. Jornal Brasileiro de Pneumologia, Ribeirão Preto, v. 34, n. 4, p. 225-229, 2008.

RODRIGUES, R.J. Information systems: the key to evidence-based health practice. Bulletin of the World Health Organization, Geneva, v.78, n. 11, p. 1344-1351, 2000.

SOARES-FILHO, A.M. O recorte étnico-racial nos sistemas de informações em saúde no Brasil: potencialidades para tomada de decisão. In: BATISTA, L.E.; WERNWCK, J.; LOPES, F. (Org). Saúde da população negra. Brasília: ABNP, 2012.

VOGT, R.L. et al. Evaluation of the timeliness and completeness of a web-based notifiable disease reporting system by a local health department. Journal of Public Health Management Practice, Greenville, v. 12, n. 6, p. 540-544, 2006.

WORLD HEALTH ORGANIZATION. Communicable disease surveillance and response systems: guide to monitoring and evaluating. Geneva, Switzerland:WHO, 2006.

YOO, H.S. et al. Timeliness of national notifiable diseases surveillance system in Korea: a cross-sectional study. BMC Public Health, London, v. 9, n. 93, p. 1-8, mar, 2009.

ZILLMER, J.G.V. et al. Avaliação da completude das informações do Hiperdia em uma unidade básica do sul do Brasil. Revista Gaúcha de Enfermagem, Porto Alegre, v. 31, n. 2, p. 240-246, jun., 2010.

Recebido para publicação em novembro de 2013

Versão final em dezembro de 2013

Conflito de interesse: não houve

Suporte financeiro: inexistente 